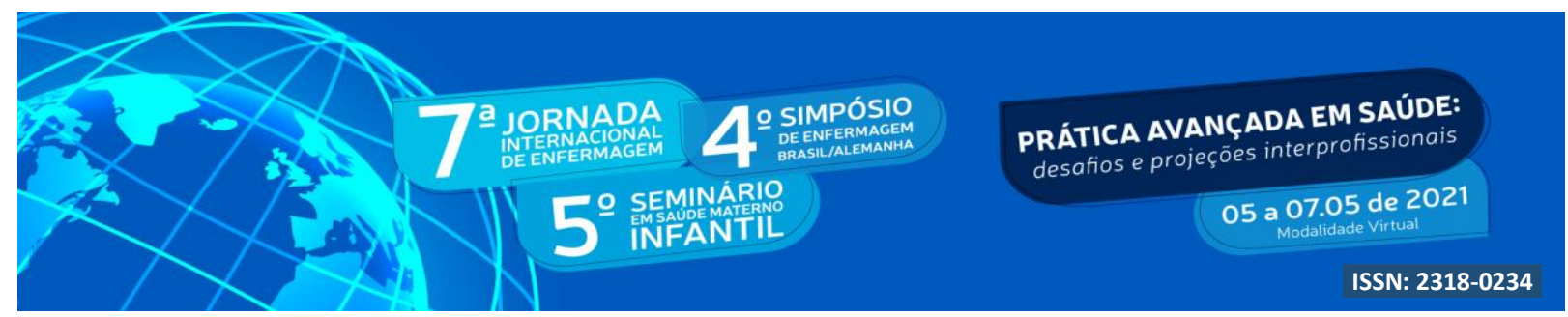

DOI: http://doi.org/10.48195/jie2021-167

\title{
IMPLEMENTAÇÃO DE AÇÕES ESTRATÉGICAS EM UMA UNIDADE CIRÚRGICO DURANTE ESTÁGIO CURRICULAR SUPERVISIONADO: RELATO DE EXPERIÊNCIA ${ }^{1}$
}

\author{
Francine Santellano Susin²; Francine Casarin³; Jessica Morais da Silva Paim4; Adriana \\ Pereira Dall'Asta ${ }^{5}$
}

\begin{abstract}
RESUMO
Introdução: A enfermagem é a profissão que possui o maior número de profissionais atuante nos serviços de saúde, sendo que estratégias que integrem teoria e prática são fundamentais para um currículo e formação desse profissional, sendo uma das mais relevantes o estágio curricular supervisionado. Objetivo: Relatar a experiência discente durante o estágio curricular supervisionado em enfermagem I em uma unidade cirúrgica. Metodologia: Se trata de um relato de experiência realizado durante a disciplina de Estágio Curricular Supervisionado em Enfermagem I do Curso de Enfermagem da Universidade Franciscana. Resultado e discussão: Ao longo do estágio, foi realizado o disgnóstico situacional e implementado ações de educação permantente para os funcionários da unidade e construção do check list do paciente e da cirurgia segura. Conclusão: Se mostrando de grande validade para o crescimento pessoal e profissional, foi desafiador e prazeroso. Podendo observar o papel na integra de um enfermeiro em uma unidade hospitalar.
\end{abstract}

Palavras-chave: Educação Permanente; Enfermagem; Hospital;

\begin{abstract}
Introduction: Nursing is the profession that has the largest number of professionals working in health services, and strategies that integrate theory and practice are fundamental to a curriculum and training of this professional, one of the most relevant being the supervised curricular internship. Objective: To report the student experience during the supervised curricular internship in nursing I in a surgical unit. Methodology: This is an experience report made during the course of Supervised Curricular Internship in Nursing I of the Nursing Course at the Franciscan University. Result and discussion: Throughout

\footnotetext{
${ }^{1}$ Relato de experiência vinculado a disciplina de Estágio Curricular Supervisionado em Enfermagem I

${ }^{2}$ Acadêmico do Curso de Enfermagem - UFN. Email: franssusin@gmail.com

${ }^{3}$ Enfermeira. Mestranda do Mestrado Ciências da Saúde e da Vida. Email: fracasarin@ @otmail.com

${ }^{4}$ Acadêmico do Curso de Enfermagem - UFN. Email: jessicamoraissilva91@ gmail.com

${ }^{5}$ Orientadora - Enfermeira - Doutora, Professora do Curso de Enfermagem - UFN. Email: adrianadallastapereira@gmail.com
} 


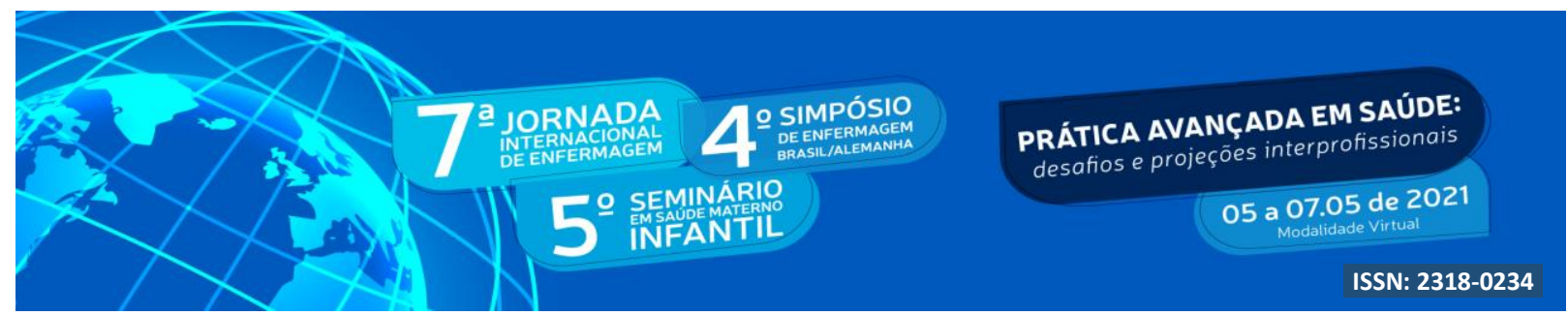

the internship, situational diagnosis was carried out and permanent education actions were implemented for the unit's employees and construction of the patient's checklist and safe surgery. Conclusion: Showing great validity for personal and professional growth, it was challenging and pleasurable. Being able to observe the role of a nurse in a hospital unit.

Keywords: Permanent Education; Nursing; Hospital;

\section{INTRODUÇÃO}

A enfermagem é a profissão que possui o maior número de profissionais atuante nos serviços de saúde, sendo também a única profissão a ficar em contato com os pacientes por 24 horas. São esses profissionais que se responsabilizam pela atenção integral à saúde de quem demanda seus cuidados. Assim, a formação dos (as) profissionais de enfermagem está diretamente ligado a construção do perfil que o mundo do trabalho espera e necessita, não apenas relacionado aos conhecimentos biológicos e técnicos, é sim sendo essencial que o acadêmico tenha um perfil crítico e que possa contribuir para a sociedade. Desta forma, as estratégias que integrem teoria e prática são fundamentais para um currículo e formação desse profissional e possam possibilite o melhor aproveitamento dos estudantes (OLIVEIRA; GRIBOSKI, 2018). De maneira que a estratégia fundamental para cumprir esse objetivo é o estágio curricular supervisionado.

Segundo as Diretrizes Curriculares Nacionais (DCN) do curso de graduação em Enfermagem, "ficam os cursos de enfermagem, obrigados a incluir no currículo o estágio supervisionado em hospitais gerais e especializados, ambulatórios, rede básica de serviços de saúde e comunidades" (MEC, 2001 pag. 5). Sendo que este é um onde se alia a teoria e prática estabelecidos ao longo dos semestres cursados. Nesta oportunidade o acadêmico precisa se posicionar como profissional possibilitando o aperfeiçoamento do aprendizado técnico, crítico e posteriormente o desenvolvimento da autonomia e segurança para exercer sua futura profissão (OLIVEIRA; GRIBOSKI, 2018).

Nesta etapa o acadêmico é inserido nos serviços de saúde, sem a presença diária do professor, mas com a supervisão de um profissional enfermeiro do local para assim poder desenvolver as competências necessárias a um profissional da categoria. Este momento gera o contato direto com profissionais, com pacientes e com conflitos e situações que surgem no dia 


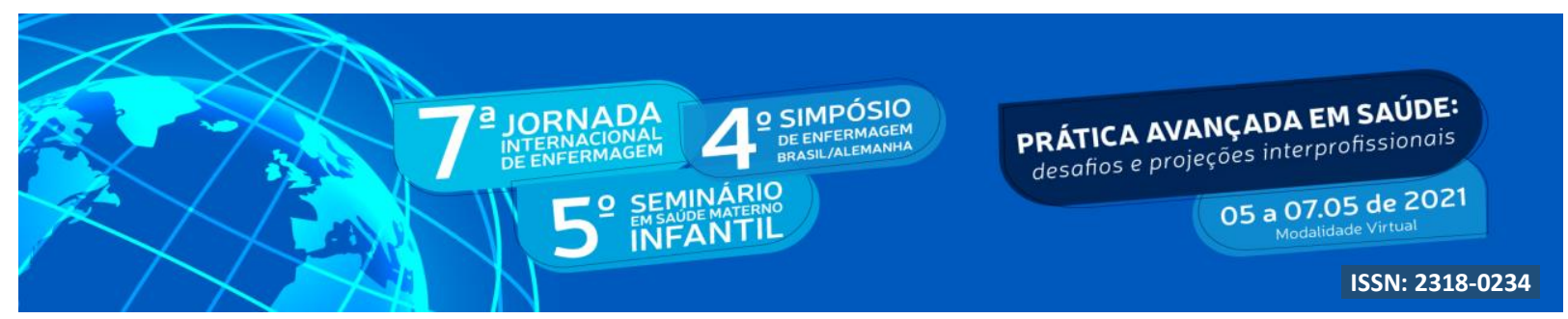

a dia do enfermeiro, com as demandas dos locais em que estarão inseridos como aprendizes e, também como profissionais habilitados, a depender do relacionamento estabelecido com a equipe. Sendo um momento rico, cheio de oportunidades de aperfeiçoamento profissional diante de situações seja na assistência direta ao paciente, no gerenciamento e gestão dos serviços prestados pela local e na construção do relacionamento interpessoal entre todos os envolvidos nas etapas do cuidado de enfermagem, fundamentais para a formação do enfermeiro (OLIVEIRA; GRIBOSKI, 2018).

\section{OBJETIVO}

Relatar a experiência discente durante o estágio curricular supervisionado em enfermagem I em uma unidade cirúrgica a cerca da implementação do check list do paciente e da cirurgia segura e da importância da educação permanente no serviço.

\section{METODOLOGIA}

O presente trabalho é de caráter descritivo, do tipo relato de experiência, realizado a partir da vivência discentes no Estágio Curricular Supervisionado em Enfermagem I (ECS I) do Curso de Enfermagem da Universidade Franciscana (UFN). Realizado no período de julho a outubro de 2020. De acordo com Gil (2008), o relato de experiência da margem para o pesquisador relatar suas experiências e vivências junto com o saber científico e assim contribuindo de forma relevante para sua área de atuação.

O local de realização do etágio foi em uma Unidade Cirúrgica pertecente a um Hospital de médio porte, no qual é um estabelecimento de saúde privado do tipo Hospital Geral que executa serviços de saúde. A mesma atende pacientes pré e pós cirúrgicos, incluído cesárias e recém nascidos. A mesma possui 6 leitos privativos e 3 quartos de semiprivativos que geravam 6 leitos. De forma que o fluxo de atendimento da unidade é por agendamento, salve cirurgias de urgência nas quais o hospital comporte. $\mathrm{O}$ recebimento de pacientes na unidade é provido de cirurgias realizadas dos tipos de traumatologia, ginecologia e obstetrícia, oftalmologia, cirurgia plástica, vascular, cirurgias gerais. Além disso, o hospital possuir 


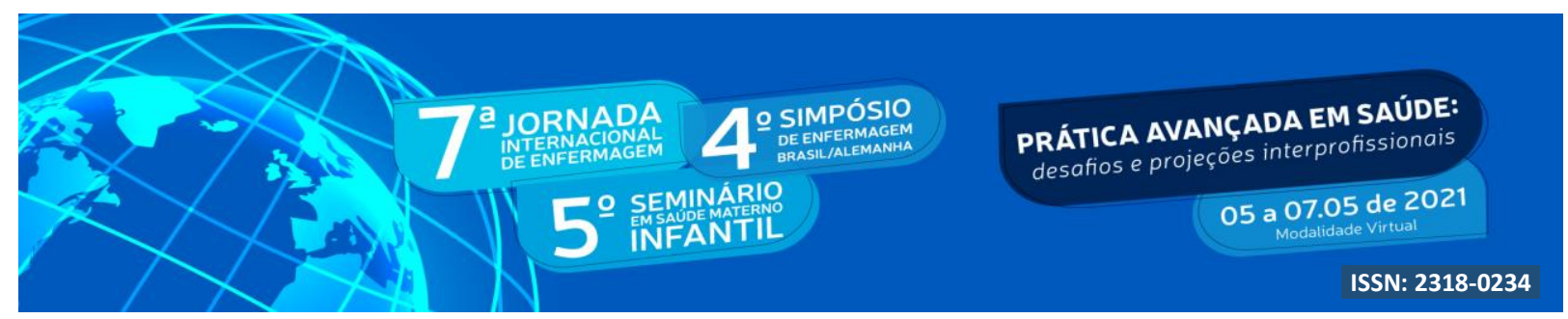

realização de exames para pacientes internados como radiologia, tomografia computadorizada, ultrassonografia, ecocardiograma, endoscopia e colonoscopia.

\section{RESULTADOS E DISCUSSÃO}

No ECSI é proposto ao discente realizar um diagnóstico situacional do serviço, onde é possível vienciar por meio da observação participante o funcionamento do serviço. A partir desse levantamento diagnóstico foi proposto, realizar uma ação estratégica de construção do check list do paciente e da cirurgia segura, o qual é preenchido com os dados do paciente recebido da internação e conferidos novamente com o paciente/acompanhante no momento da internação do paciente no quarto, e realizado algumas orientações. Também nesse momento foi proposto realizar encontros de Educação permanente sobre algumas temáticas que são importantes pro cuidado ao paciente.

Entre a equipe de enfermagem o processo comunicativo é algo fundamental para garantir a excelência do trabalho, para a qualidade da assistência e também o bom relacionamento entre os profissionais e os pacientes. Reforça-se ainda que o diálogo cria uma aproximação entre as pessoas, gerando um contato mais próximo, uma relação de integração de culturas, uma troca de vivências e experiências. Sendo que, uma relação de comunicação eficiente entre todos os membros da equipe de enfermagem reflete para que as inter-relações estabelecidas no trabalho delimitem melhor a assistência ao paciente de forma humanizada, sendo importante respeitar cada profissional na sua individualidade (BROCA; FERREIRA, 2012).

Nesse sentido, a partir da observação da necessidade de realização de procedimentos com segurança, uma vez que é possivel no check list deixar registrado e comunicar a equipe quando um paciente esta com a pressão arterial sistêmica alterada e por meio do check list é possível saber se existe possui comorbidades relacionadas a pressão arterial, ou somente ansiedade por causa da cirurgia ser realizada e assim intervir com a escuta ativada dos paciente e os procedimentos para acalma-lo.

Posteriormente realizadas todas ações necessárias antes da realização da cirurgia para conferência do membro correto e dos materiais necessários para realização da cirúrgica 


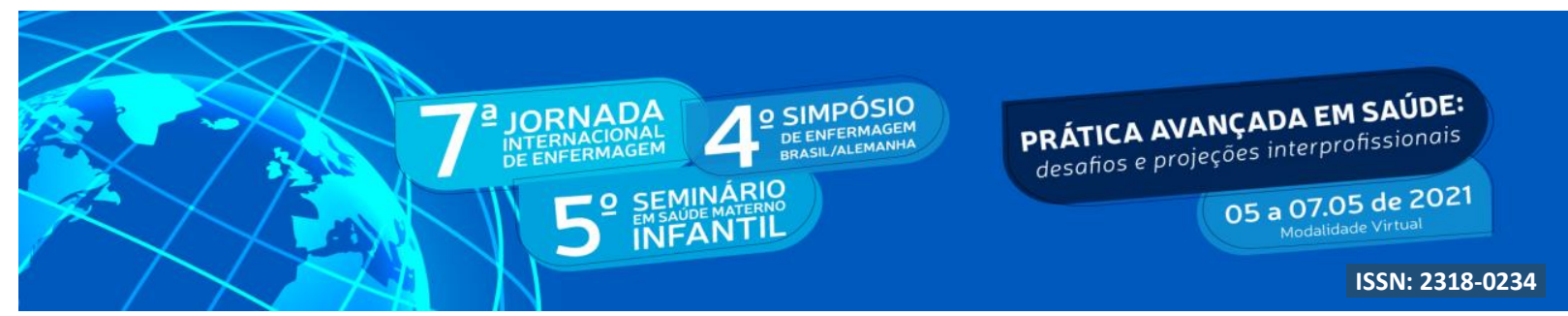

(contato com médico e centro de esterilização de material), o paciente então é encaminhado no horário pré agendado ao Centro cirúrgico. Nestas intervenções, pode-se perceber o quanto os check list realizado da forma correta são fundamentais para que o processo ocorra da forma correta.

Observa-se que, um dos temas debatidos pela Organização Mundial de Saúde (OMS) é a segurança do paciente cirúrgico sendo denominada: "Cirurgia Segura Salva Vidas", com o objetivo de reduzir as ocorrências de danos e definir padrões de segurança a serem empregados em diversos países. Em 2008, o Ministério da Saúde do Brasil aderiu à campanha já citada tendo como objetivo principal a adoção, pelos hospitais, de uma lista de verificação padronizada, para assessorar as equipes cirúrgicas na diminuição de danos e erros ao paciente (SILVA, et al. 2017).

Ressaltando-se assim que, dentre as ações de gestão do enfermeiro está a tomada de decisão, sendo possível destacar aquelas ações relacionadas à segurança do paciente, nas quais podem diminuir aos eventos adversos, as complicações cirúrgicas e a detecção precoce de intercorrências no período pré e pós-operatório. Com isso, o desenvolvimento de ferramentas que forneçam informação e gerem segurança ao pacientes, está o checklists, no qual, promovem a identificação precoce de problemas e erros que podem ocorrer na assistência hospitalar. Sendo que a Organização Mundial da Saúde aprova a utilização de ferramenta como o cheklist para uma das formas de melhorar a segurança do paciente, observando diversos estudos relacionados comprovando a sua eficiência e eficácia (FERREIRA, et al., 2019).

O checklist é uma ferramenta para ser utilizada em todos os hospital, independentemente do seu grau de complexidade, tendo como objetivo auxiliar as equipes de cirurgia a seguirem de forma sistemática passos críticos de segurança, tendo o foco a segurança na assistência cirúrgica, podendo também promover a melhor comunicação e interação multiprofissional. É fundamental destacar que durante a aplicação do Checklist a equipe de saúde tenha integração e interação nos processo. Sendo uma ferramenta não somente de checagem, mas também um elemento facilitador na assistência ao paciente, comunicação entre a equipe e segurança do paciente (SILVA, et al. 2017). Mostrando o quando é importante a utilização desta ferramenta para segurança do paciente e que é 


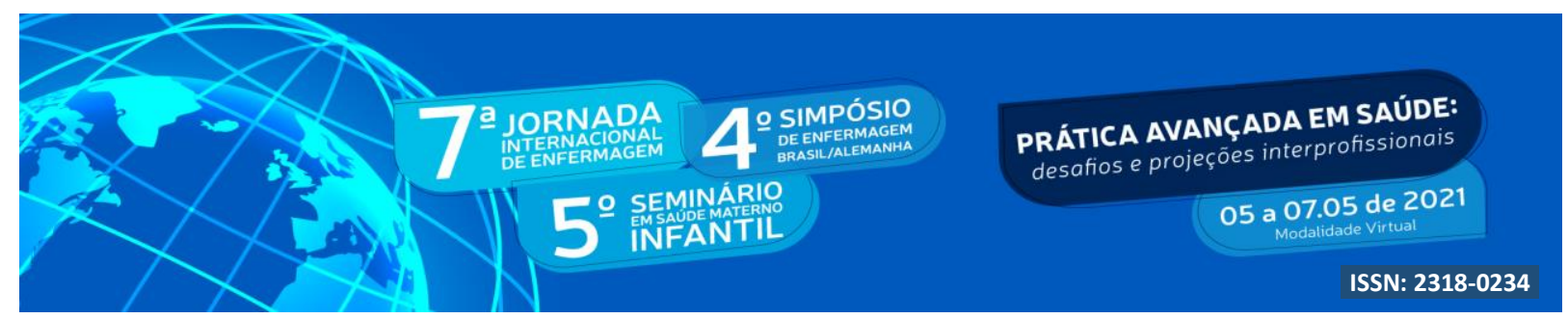

imprescindível para que seja bem aplicada a comunicação entre a equipe, reforçando o que já foi citado.

Durante o estágio, através da observação da unidade durante os primeiros dias de estágio, acompanhamento de reunião onde sempre ocorre uma educação permanente e conversas diárias com a enfermeira e realização do diagnóstico situacional, em consenso com a enfermeira foi realizado a educação permanente em reunião de equipe que já estava agendada. A partir disso, observou-se todos processos gerenciais da unidade e escolhi trabalhar com a equipe a temática de Lesão por Pressão (LPP). Essa escolha deu-se pois percebi com o passar dos dias que a unidade recebe vários pacientes idosos, nos quais possuem a pele frágil devido a idade e também pacientes providos de cirurgias de traumatologia, que ficam um pouco mais privados ao leito, sem poder mexer tanto devido ao tipo de cirurgia e assim esses dois casos de alta incidência na unidade podem ter o risco de gerar Lesões por Pressão. Ainda se ressalta que se observarmos e cuidarmos desde o início as LPP podemos evitar, as vezes não que ocorra, mas que não chegue ao um estágio mais grave. Fato esse que motivou a minha ideia de tema para a realização de uma educação permanente.

Desta forma foi exposto para enfermeira essa visão e proposto que na próxima reunião de equipe pudesse ser falado sobre os tipos de lesão por pressão (LPP) e quais os cuidados os técnicos em conjunto com os enfermeiros poderiam realizar para prevenir as LPP. Assim foi realizada uma pesquisa sobre o assunto, realizando uma descrição dos estágios, como a pele se apresentava em cada uma, qual camada era atacada, quando poderia ser visível gordura e posteriormente músculos, tendões e osso. Foi realizado ainda um esquema com desenhos sobre isso para facilitar a visualização e poder deixar no mural da unidade para tirar dúvidas sempre que houver.

A educação permanente acerca do tema: risco de lesão por pressão, foi realizada entre o turno da manhã e tarde (13 à 14h) com toda equipe da unidade, em uma roda de conversa. Inicialmente me senti um pouco nervosa, a enfermeira me deu mais segurança ao desenrolar e consegui realizar com sucesso, fui parabenizada por todas as partes e tive o feedback das técnicas que seria sim, muito útil para elas e que poderiam melhorar o cuidado com os pacientes da unidade.

Contextualiza-se assim que a os problemas enfrentados no dia a dia pelos profissionais podem ser fundamentos para o exercício da educação permanente, que se desenvolve a partir 


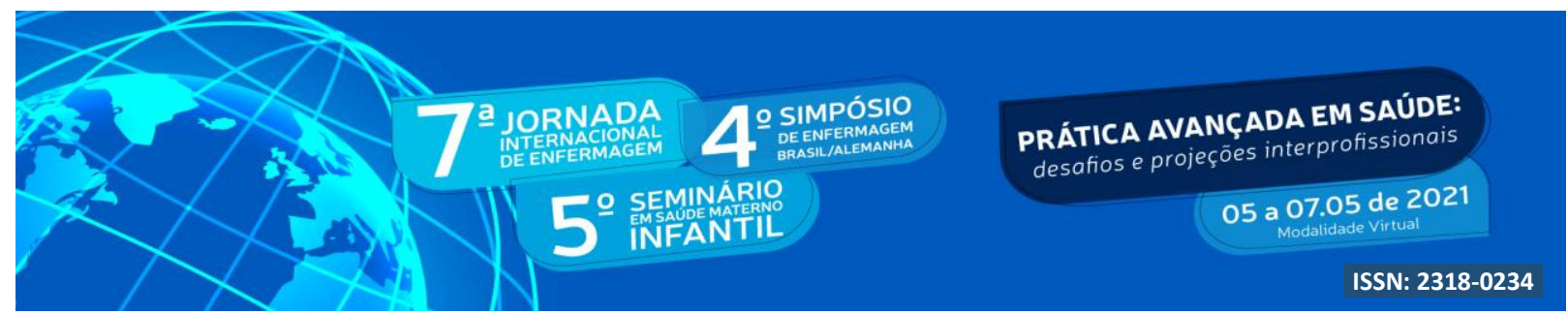

das necessidades e problemas presentes em um serviço de saúde. Assim, deve-se pensar que as ações educacionais podem ter como objeto a realidade, porém, é necessário aprender a ler a realidade para que se possa transformá-la e após utilizar-se de aportes teóricos que ampliem o olhar crítico dos trabalhadores, profissionais e gestores nos serviços de saúde. Sendo assim, caracteriza-se a educação permanente, demandada pelos sujeitos que estão mergulhados no cotidiano e a complexidade de seus problemas, necessitando mudanças nos modos de se realizar a atenção à saúde. Assim, a educação permanente é uma ferramenta de grande relevância para a gestão, para auxiliar nas melhorias dos cuidados aos pacientes e a capacitação dos seus profissionais (CAMPOS; SENA; SILVA, 2017).

Ainda durante o estágio, foi observado que existia alguns modelos de evolução em uma pasta das normas e rotinas da unidade, na qual no início do estágio a enfermeira mostrou, notando assim que alguns estavam desatualizado e percebido que, quando a unidade estava com a lotação cheia se torna um pouco complicado escrever toda as evoluções com todos os componentes necessário, as vezes a carga em cima do enfermeiro, como já citado é alta e precisamos dar conta não apenas da tarefa de cuidado, mas a gestão e gerencia da unidade bem como dos profissionais, percebido assim, que estes modelos estando no computador e atualizados poderiam contribuir para um processo mais dinâmico do enfermeiro ao registrar evoluções, dentre elas estão: evolução de internação, evolução diária completa, evolução puérpera diária, evolução do RN, evolução de curativos e evolução de alta.

Solicitado para enfermeira se fosse possvel digitar todos eles no computador e posteriormente atualizar eles de acordo com a vivência do dia a dia. Desta forma, foi ajustado, sendo que eles eram evoluções gerais que o técnico e o enfermeiro poderiam usar, pensando mais além ao final das duas evoluções mais importantes a diária completa e a da puérpera completa foi acrescenado ao final a parte específica do enfermeiro, com todo o exame físico privativo a ao enferemeiro.

Após essa construção, foi percebido que em dias de grande demanda do pessoal se tornava sim, bem útil para utilização e a enfermeira estava também conseguindo usa-la, ocorreu até o fato de uma acadêmica de outra unidade solicitar a versão que eu tinha feito para ela imprimir para utilizar o que pudesse na unidade que ela estava estagiando, me senti nesse momento lisonjeado e que realmente foi útil a ideia. Ao final a enfermeira informou que na 


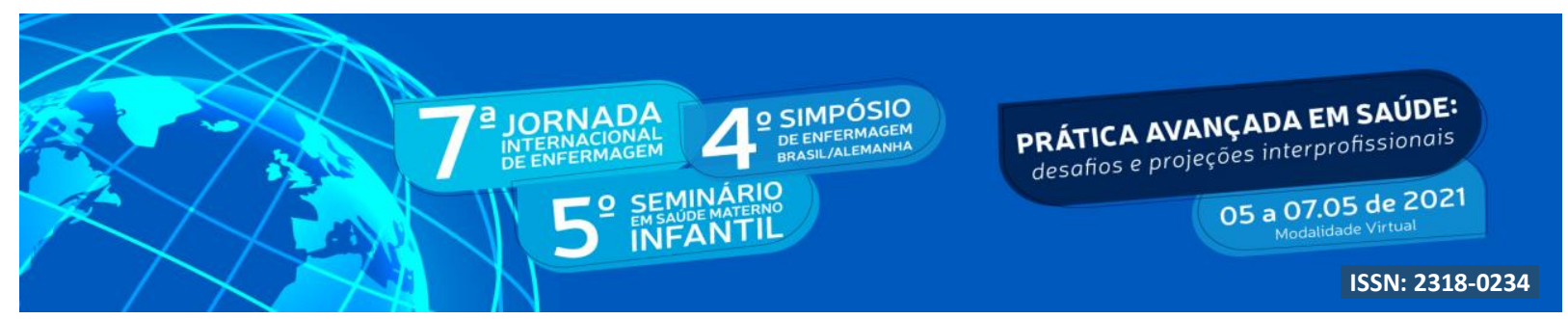

próxima revisão anual que é feita no manual de procedimentos e rotina irá repassar para responsável para aprovação dos mesmos para incluir o modelo que foi atualizado.

Os registros de enfermagem englobam uma forma de comunicação escrita das informações pertinentes ao paciente e aos cuidados recebidos durante todo o processo de assistência realizados pela equipe de enfermagem. Compreendem elementos essenciais na documentação do cuidado integral, considerando que, quando redigidos de forma correta, possibilitam a comunicação entre todos envolvidos nesse processo do cuidado. Ainda percebe-se que servem de base para a elaboração do plano assistencial adequado ao paciente; fornecem subsídios para a avaliação da assistência prestada; auxiliam no acompanhamento da evolução do paciente; favorecem a auditoria de enfermagem; fornecem o respaldo legal que o profissional necessita e ainda podem colaboram com o ensino e pesquisa em enfermagem (BRAGAS, 2015).

Por fim, na última reunião de equipe que participei, em meio ao momento de exposição dos técnicos de enfermagem de problemas que tiveram durante o mês, surgiu a demanda de que, as vezes as grávidas que irão realizar parto Cesária chegam na unidade sem algumas coisas básicas necessárias para ela e para o bebê e foi observado pelas técnicas de enfermagem que muitas veze elas ligam para secretária do hospital que não sabe informar direito o que é necessários, alguns momentos elas conseguem transferir a ligação para unidade e falar com algum dos profissionais que sabe realizar essa orientação, porem existe o caso de os profissionais estarem ocupados e que daí as mesmas repassas as informações e pro algumas vezes incompletas. Surgiu assim a ideia de realizar um folder, para deixar no balcão da secretária com essas informações, para que assim quando alguém ligasse para pergunta elas mesmas pudesse passar essa informação de forma completa. Assim com a autorização da enfermeira confeccionei esse folder com todas as informações necessárias e para a gravida e para o bebê e após aprovação da enfermeira foi disponibilizado o mesmo para secretária do hospital.

\section{CONCLUSÃO}

Este estágio foi de extrema importância para o meu crescimento pessoal e profissional, foi desafiador e prazeroso. Foi possível visualizar a gestão e gerência da enfermeira em uma 


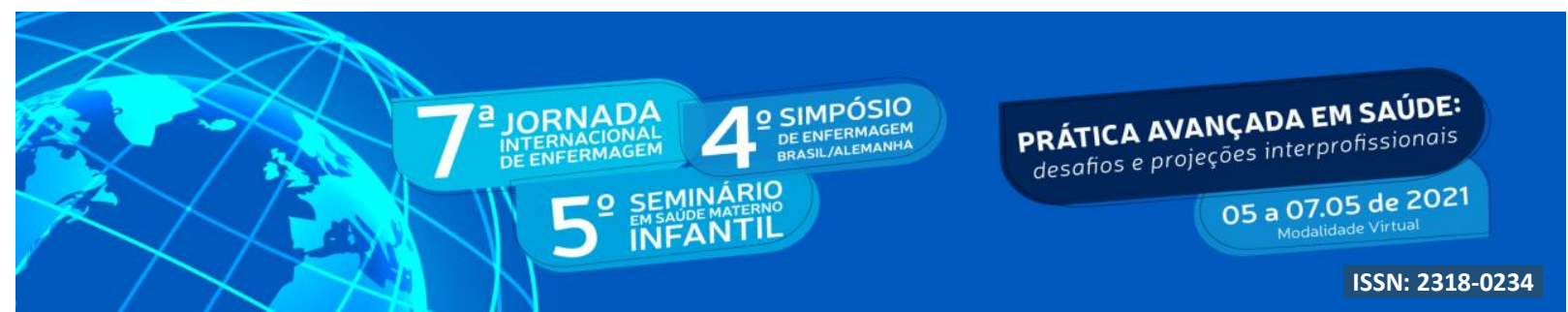

unidade hospitalar, com todos os problemas que podem surgir e a solução que se pode tomar a cada momento, podendo futuramente aplicar isso em minha vida profissional. Visualizei de forma eficaz o trabalho em equipe e uma boa comunicação entre os seus membros para que o cuidado seja de qualidade, sendo que com certeza quero seguir o exemplo que vivencie de uma enfermeira líder que entende sua equipe e consegue se comunicar com ela.

Durante a realização das atividades propostas e desenvolvidas no ECS I, foi possível desenvolver as habilidades necessárias para como futuro profissional e refletir sobre o processo de trabalho do enfermeiro.

\section{REFERÊNCIAS}

BRAGAS, L. Z. T. A importância da qualidade dos registros de enfermagem para gestão em saúde: estudo em hospital na região noroeste do rs. Trabalho de conclusão de curso de Especialização de Gestão em Saúde. Escola de Administração/UFRGS - Universidade Aberta do Brasil (UAB). Porto Alegre, 2015.

BROCA, P. V.; FERREIRA, M. A. Equipe de enfermagem e comunicação: contribuições para o cuidado de enfermagem. Rev. bras. enferm., Brasília, v. 65, n. 1, p. 97-103, fev. 2012.

CARLESI, K. C. et al. Ocorrência de incidentes de Segurança do Paciente e Carga de Trabalho de Enfermagem. Rev. Latino-Am. Enfermagem, Ribeirão Preto, v. 25, e2841, 2017 .

CAMPOS, K. F. C.; SENA, R. R.; SILVA, K. L. Educação permanente nos serviços de saúde Escola Anna Nery Revista de Enfermagem, v. 21, n. 4, p. 1-10, 2017.

DALRI, R. C. M. B.; et al. Carga horária de trabalho dos enfermeiros e sua relação com as reações fisiológicas do estresse. Rev. Latino-Am. Enfermagem, v. 22, n. 6, p. 959-965, nov.-dez. 2014.

FERREIRA, N. C. B., et al. Checklist de Cirurgia Segura: Conhecimento e Utilização do Instrumento na Perspectiva dos Técnicos de Enfermagem. Revista de Enfermagem do

Centro Oeste Mineiro. v. 9. 2019. 


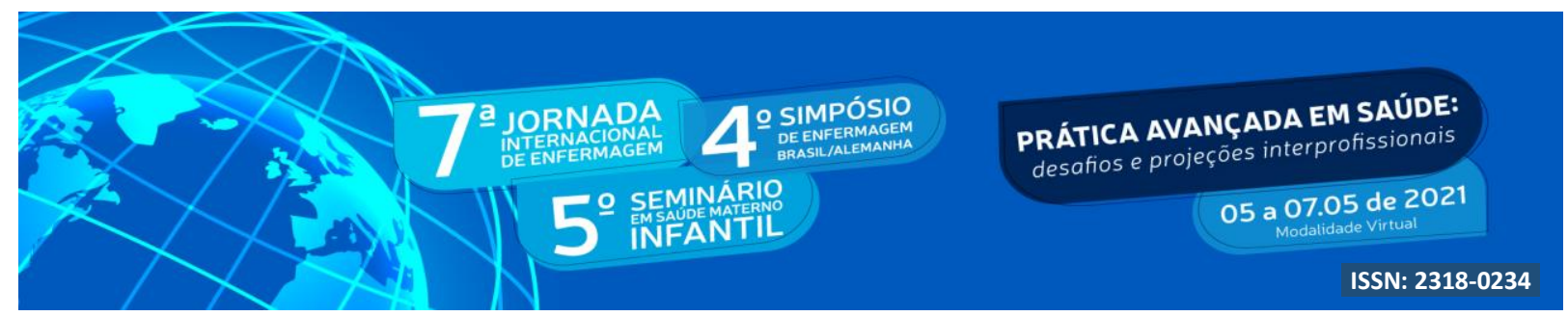

GAUER, A. P. M. et al. Ações de reorientação da formação profissional em Fisioterapia: enfoque sobre cenários de prática. Interface (Botucatu), Botucatu, v. 22, n. 65, p. 565576, abr. 2018.

MEC. Ministério da Educação. Diretrizes curriculares nacionais do curso de graduação em enfermagem. 2001 Disponível em < http://portal.mec.gov.br/cne/arquivos/pdf/Enf.pdf>. Acesso em 9 de out. 2020.

OLIVEIRA, A. P. A.; URBANETTO, J. S.; CAREGNATO, R. C. A. Adaptação transcultural e validação da national early warning score 2 para o brasil. Revista enfermagem UFPE on line, Recife, v. 12, n. 11, p. 3154-3157, nov. 2018.

OLIVEIRA, W. G., GRIBOSKI, C. M. O estágio supervisionado na formação do enfermeiro revisão integrativa. Trabalho de conclusão de curso. 2018. Disponível em: $<$ https://www.bdm.unb.br/bitstream/10483/24553/1/2018_WalquiriaGomesDeOliveira_tcc.pd f>. Acesso em 9 de out. 2020.

SILVA, E. F. M.; et al. Conhecimento dos profissionais da saúde sobre checklist de cirurgia segura. Arq. Ciênc. Saúde, v. 24, n. 3, p. 71-78, jul-set 2017. 